\title{
MONITORING THE DEFORMATION OF HIGH-RISE BUILDINGS IN SHANGHAI LUIJIAZUI ZONE BY TOMO-PSINSAR
}

\author{
L. F. Zhou ${ }^{1 *}$, P. F. Ma ${ }^{2}$, Y. Xia ${ }^{1}$, C. H. Xie ${ }^{1}$ \\ ${ }^{1}$ Changshu Institute of Technology, Changshu, China - zhoulifan_rs@163.com \\ ${ }^{2}$ Institute of Space and Earth Information Science, The Chinese University of Hong Kong, Hong Kong, China - idowman@ucl.ac.uk
}

KEY WORDS: High-rise buildings, Lujiazui Zone, Thermal expansion, Creep and shrinkage, Tomo-PSInSAR

\begin{abstract}
:
In this study, we utilize a Tomography-based Persistent Scatterers Interferometry (Tomo-PSInSAR) approach for monitoring the deformation performances of high-rise buildings, i.e. SWFC and Jin Mao Tower, in Shanghai Lujiazui Zone. For the purpose of this study, we use 31 Stripmap acquisitions from TerraSAR-X missions, spanning from December 2009 to February 2013. Considering thermal expansion, creep and shrinkage are two long-term movements that occur in high-rise buildings with concrete structures, we use an extended 4-D SAR phase model, and three parameters (height, deformation velocity, and thermal amplitude) are estimated simultaneously. Moreover, we apply a two-tier network strategy to detect single and double PSs with no need for preliminary removal of the atmospheric phase screen (APS) in the study area, avoiding possible error caused by the uncertainty in spatiotemporal filtering. Thermal expansion is illustrated in the thermal amplitude map, and deformation due to creep and shrinkage is revealed in the linear deformation velocity map. The thermal amplitude map demonstrates that the derived thermal amplitude of the two highrise buildings both dilate and contract periodically, which is highly related to the building height due to the upward accumulative effect of thermal expansion. The linear deformation velocity map reveals that SWFC is subject to deformation during the new built period due to creep and shrinkage, which is height-dependent movements in the linear velocity map. It is worth mention that creep and shrinkage induces movements that increase with the increasing height in the downward direction. In addition, the deformation rates caused by creep and shrinkage are largest at the beginning and gradually decrease, and at last achieve a steady state as time goes infinity. On the contrary, the linear deformation velocity map shows that Jin Mao Tower is almost stable, and the reason is that it is an old built building, which is not influenced by creep and shrinkage as the load is relaxed and dehydration proceeds. This study underlines the potential of the Tomo-PSInSAR solution for the monitoring deformation performance of high-rise buildings, which offers a quantitative indicator to local authorities and planners for assessing potential damages.
\end{abstract}

\section{INTRODUCTION}

Lying on the east bank of Huangpu River and facing the Bund, Lujiazui Finance and Trade Zone (Lujiazui Zone for short) in Shanghai city, China, occupies 28 square kilometers $(10.8$ square miles) and is studded with around 100 high-rise buildings. Many are landmark buildings of Shanghai, making the area a great place to experience the modern flavor of this metropolis. The surface motion and structural instability of the high-rise buildings can significantly influence the serviceability of buildings and should be constantly inspected carefully.

During the last twenty years, the interferometric synthetic aperture radar (InSAR) technology has developed into a powerful geodetic tool to detect large scale land deformation with high accuracy and large spatial coverage, especially the Persistent Scatterer SAR Interferometry (PSInSAR) technology has proven to be an effective tool to detect ground deformation in urban built environments (Ferretti et al., 2000). The ground subsidence rates of the central area of Shanghai have been obtained by PSInSAR technique in recent years. Perissin et al. (2012) extracted land subsidence in Shanghai by using 33 Cosmo-SkyMed SAR images with the SARPROZ software, and concentrated on tracking the paths of newly excavated subway tunnels, which allows one to analyze the transverse motion profiles over metro tunnels and predict possible building damage. Jiang et al. (2016) gave a time-series analysis of a high-resolution TerraSAR-X satellite image stack acquired from September 2011 to October 2012 by employing a modified PSInSAR technique to retrieve the mean deformation velocity and time series of surface deformation at Pudong International Airport. Wu and Hu (2017) applied the PSInSAR method to extract ground subsidence using $\mathrm{X}$-band TerraSAR-X images along the Shanghai Maglev Zone. The results show that persistent scatters (PS) are distributed on the rail of the maglev, and the subsidence rates of these PSs points are mostly less than $3 \mathrm{~mm} /$ year.

However, it is still a challenge to apply PSInSAR technique to effectively monitor the deformation of high-rise buildings in the central business district for three major reasons: first, the highrise buildings often cause the mixing of reflections from building facades and ground targets. Without considering double PSs, several buildings would not match the classic phase model for PSs and would be interpreted as noisy reflections. Second, high-rise built environment, e.g., Shanghai Lujiazui Zone, is with cloudy and rainy weather, where there is much uncertainty when removing the atmospheric phase screen (APS) in spatiotemporal filtering. Third, thermal expansion repeats periodically with seasonal changes in temperature throughout

\footnotetext{
* Corresponding author
} 
the entire lifetime of the buildings. If we do not remove thermal expansion, the classic phase model for PSs would fail and highrise buildings would appear as noisy targets. Considering the limitations of conventional PSInSAR technique, a Tomographybased Persistent Scatterers Interferometry (Tomo-PSInSAR) technique is proposed by (Ma and Lin, 2016), which integrates merits from PSInSAR (Ferretti et al., 2000) and SAR tomography (Ma et al., 2015). In this study, we utilize the Tomo-PSInSAR approach for monitoring the deformation performances of the two landmark skyscrapers in Shanghai Luijiazui Zone.

\section{STUDY SITE AND SAR DATA USED}

\subsection{Study Area}

Shanghai Lujiazui Zone is located in the Pudong New District on the eastern bank of Huangpu River. It forms a peninsula on a bend of the Huangpu River, which turns from flowing north to flowing east. Currently, this district includes many high-rise buildings. Among them, the two landmark buildings are selected for analysis in this study: Jin Mao Tower and Shanghai World Financial Center (SWFC). Jin Mao Tower is 421 meters and is the third tallest building only to Shanghai Tower and SWFC in Shanghai. It is an old building which was completed in 1999 long time before the beginning time of the acquisitions. SWFC is a newly built building and was complete in the second half of 2008, and the height is approximately $490 \mathrm{~m}$, being the second tallest skyscraper in mainland China. The major parameters of the two landmark buildings are listed in Table 1.

\begin{tabular}{|l|l|l|c|c|}
\hline $\begin{array}{l}\text { Building } \\
\text { names }\end{array}$ & Image & $\begin{array}{l}\text { Height } \\
(\mathrm{m})\end{array}$ & $\begin{array}{l}\text { Number of } \\
\text { floors }\end{array}$ & Year \\
\hline $\begin{array}{l}\text { Shanghai } \\
\text { World } \\
\text { Financial } \\
\text { Centre } \\
\text { (SWFC) }\end{array}$ & & 492 & 101 & 2008 \\
$\begin{array}{l}\text { Jin Mao } \\
\text { Tower }\end{array}$ & & 421 & 93 & 1998 \\
& & & & \\
\hline
\end{tabular}

Table 1. Major parameters of the two landmark buildings.

\subsection{TerraSAR-X and Temperature Data}

For the purpose of the study, 31 TerraSAR-X ascending Stripmap SAR acquisitions in $\mathrm{HH}$ polarization, acquired from December 2009 to February 2013, are utilized for monitoring the two high-rise buildings in Shanghai Lujiazui Zone. Pixel samplings in azimuth and range direction are $1.71 \mathrm{~m}$ and $0.91 \mathrm{~m}$ respectively, approximately equal to a ground resolution of $3 \mathrm{~m}$, considering a central incidence angle of $22.7^{\circ}$. Daily temperature data (https://www.wunderground.com/), collocated to SAR acquisitions, are obtained for the calculation of thermodynamics of building materials. The interferometric parameters of TerraSAR-X acquisitions in conjunction with the corresponding temperature data are listed in Table 2. The distributions of the temporal baseline and spatial normal baseline are depicted in Figure 1 with the image acquired of 2011-10-27 as the reference image. The two skyscrapers in Shanghai Luijiazui Zone are displayed based on the averaged TerraSAR-X intensity image as shown in Figure 1. Among them, Jin Mao Tower is indicated by a yellow rectangle and SWFC is indicated by a red rectangle.

\begin{tabular}{|c|c|c|c|}
\hline No. & Acquisitions & Bperp (m) & Temp. $\left({ }^{\circ} \mathrm{C}\right)$ \\
\hline 1 & $2009-12-14$ & 41.1201 & 10 \\
\hline 2 & 2010-01-16 & 52.95 & 6 \\
\hline 3 & 2010-02-18 & -90.69 & 4 \\
\hline 4 & 2010-03-23 & -16.16 & 8 \\
\hline 5 & 2010-04-25 & -103.01 & 14 \\
\hline 6 & $2010-05-28$ & 48.31 & 18 \\
\hline 7 & 2010-06-30 & 4.93 & 31 \\
\hline 8 & 2010-08-02 & -13.47 & 32 \\
\hline 9 & 2010-09-04 & -60.65 & 29 \\
\hline 10 & 2010-10-07 & -137.54 & 21 \\
\hline 11 & 2010-11-09 & 58.91 & 14 \\
\hline 12 & 2010-12-12 & 350.12 & 12 \\
\hline 13 & 2011-10-05 & -104.15 & 21 \\
\hline 14 & 2011-10-27* & 0 & 18 \\
\hline 15 & 2011-11-18 & -74.76 & 21 \\
\hline 16 & 2012-01-01 & -62.80 & 6 \\
\hline 17 & 2012-01-23 & 74.95 & 1 \\
\hline 18 & 2012-02-14 & -122.91 & 6 \\
\hline 19 & 2012-03-07 & -33.04 & 11 \\
\hline 20 & 2012-04-20 & -17.82 & 18 \\
\hline 21 & 2012-05-12 & -55.23 & 22 \\
\hline 22 & 2012-06-03 & 31.75 & 21 \\
\hline 23 & $2012-06-25$ & -213.45 & 24 \\
\hline 24 & 2012-07-17 & 135.52 & 26 \\
\hline 25 & 2012-08-30 & 127.54 & 27 \\
\hline 26 & 2012-09-21 & -50.42 & 23 \\
\hline 27 & 2012-10-13 & 83.93 & 22 \\
\hline 28 & 2012-11-26 & -193.85 & 8 \\
\hline 29 & $2012-12-18$ & -43.03 & 2 \\
\hline 30 & 2013-01-09 & -69.59 & 4 \\
\hline 31 & 2013-02-11 & 14.66 & 6 \\
\hline
\end{tabular}

Table 2. Interferometric parameters of TerraSAR-X acquisitions in conjunction with the corresponding temperature data. The acquisition of 2011-10-27 (indicated by '*' symbol) is selected as the reference image for the interferogram generation to minimize spatial-temporal decorrelation.

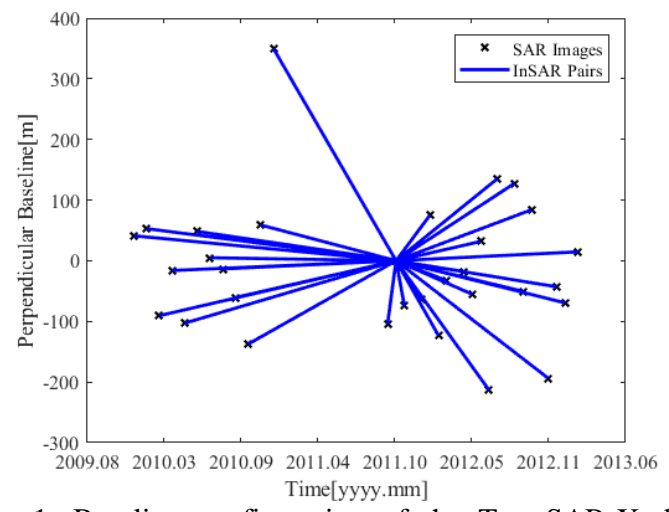

Figure 1. Baseline configuration of the TerraSAR-X dataset used in this study. 


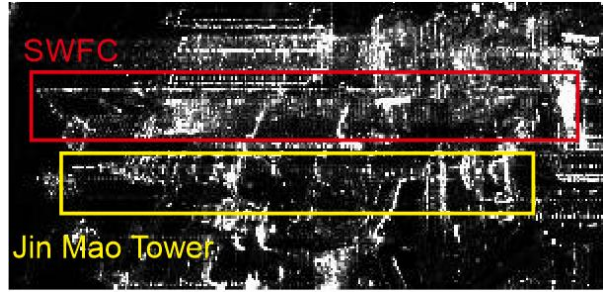

Figure 2. The two skyscrapers in Luijiazui Zone are displayed based on the averaged TerraSAR-X intensity image. The red rectangle in the top figure denotes SWFC, and the yellow rectangle denotes Jin Mao Tower.

\section{METHODOLOGY}

\subsection{Extended 4-D SAR Phase Model}

$N$ coregistered interferograms are generated using the star-graph approach based on the reference image selected. Orbit parameters are applied for the removal of flat-earth, resulting in interferograms whose unwrapped phase $\varphi_{i}, i=1, \ldots, N$, comprises four components:

$$
\varphi_{i}=\left(\varphi_{\text {ele }}\right)_{i}+\left(\varphi_{\text {def }}\right)_{i}+\left(\varphi_{\text {APS }}\right)_{i}+\left(\varphi_{\text {dec }}\right)_{i}
$$

where $\varphi_{\text {ele }}$ is the elevation phase contribution, $\varphi_{\text {def }}$ is the deformation phase contribution, $\varphi_{\text {APS }}$ is the APS phase contribution, and $\varphi_{\mathrm{dec}}$ is the decorrelation noise phase contribution. $\varphi_{\text {ele }}$ in (1) can be modelled by

$$
\left(\varphi_{\text {ele }}\right)_{i}=2 \pi \varepsilon_{i} s, \varepsilon_{i}=2 B_{i} / \lambda R
$$

where $B$ is the normal baseline with respect to the reference image, $\lambda$ is the wavelength, $R$ is the slant range between target and sensor, and $s$ is the slant elevation, which can be converted to vertical height $h=s \cdot \sin (\theta)$. $\varphi_{\text {def }}$ in (1) can be expressed by an extended motion (linear motion plus thermal expansion) model (Ma et al., 2015), given by

$$
\left(\varphi_{\text {def }}\right)_{i}=2 \pi\left(\eta_{i} v+\tau_{i} k\right), \eta_{i}=2 t_{i} / \lambda, \tau_{i}=2 T_{i} / \lambda
$$

where $t$ and $T$ are temporal and thermal baseline, respectively; $v$ and $k$ are linear deformation rate and thermal amplitude respectively. Note that thermal expansion, creep and shrinkage are two long-term movements that occur in high-rise buildings with concrete structures. Therefore, we use an extended 4-D SAR phase model, formulated as (Ma and Lin, 2016)

$$
y_{i}=\iiint_{\Delta s \Delta v \Delta k} \gamma(s, v, k) \exp \left[-j \varphi_{n}(s, v, k)\right] d s d v d k
$$

where $y_{i}$ is the Single Look Complex (SLC) pixel value. $\gamma(s, v, k)$ is the target reflectivity profile in the space of $(s, v, k) . \Delta s, \Delta v$, $\Delta k$ is the range of the expected elevation, linear motion and phase-to-temperature sensitivity value respectively. Unlike PSInSAR which assumes that each pixel contains one PS at most, Tomo-PSInSAR synergistically analyzes the amplitude and phase of points based on the assumption of more than one PS in one pixel. That is to say, there is more than one value in the reconstructed $\gamma$. Consequently, we reconstruct the tomography $\gamma$ for the motion, height and thermal amplitude parameters using spectral estimators, given by

$$
\begin{gathered}
\hat{\gamma}(s, v, k)=\left(\frac{\left|a(s, v, k)^{H} y\right|}{\|a(s, v, k)\|_{2}\|y\|_{2}}\right) \\
a(s, v, k)=\left[\begin{array}{c}
\exp \left(j 2 \pi\left(\varepsilon_{1} s+\eta_{1} v+\tau_{1} k\right)\right) \\
\cdots \\
\exp \left(j 2 \pi\left(\varepsilon_{N} s+\eta_{N} v+\tau_{N} k\right)\right)
\end{array}\right]
\end{gathered}
$$

where $(\cdot)^{H}$ stands for the transpose and conjugate operation, and $\bullet_{2}$ is 2-norm. By identifying the peaks in the normalized tomographic magnitude (NTM), we can determine how many PSs interfere in the pixel. The spectral estimator (5), e.g., beamforming in our study, is used to reconstruct tomography. Beamforming differs from the periodogram used in PSInSAR approach in that it exploits amplitude information in addition to phase information. It is worth mention that Tomo-PSInSAR approach extends PSInSAR approach by relaxing the assumption that each pixel contains only one PS, and can be therefore effective in urban built environments.

\subsection{Two-tier Network Strategy}

We apply a two-tier network strategy to detect single and double PSs with no need for preliminary removal of the APS in the study area. In the first-tier network, single PS candidates are initially selected using the amplitude dispersion criterion (Ferretti et al., 2000), e.g., a threshold of 0.28 in this study. After a Delaunay triangulation is applied to connect single PS candidates, we employ the beamforming to reconstruct the tomography for the PS candidates according to (5). If the maximum NTM is larger than a given threshold, e.g., 0.75 in this study, we consider that the two single PSs candidates at the arc are true single PSs and preserve the arc. Then, we use an Mestimator (Huber, 1964) for the estimation of relative parameters (height, linear motion rates, and thermal amplitude) at arcs. An advantage of such an approach is that atmospheric artifacts can be ignored. After that, the motion, height, and thermal amplitude estimates are integrated through network adjustment to refer measurements to a common reference point. For the potentially ill-adjusted regulate problem, we use a ridge estimator (Ma and Lin, 2016) for network adjustment.

In the second-tier network, double PS and the remaining single PS points are extracted using the local maximum ratio (Ma and Lin, 2016) and amplitude dispersion criterion (Ferretti et al., 2000) respectively, referring to single PSs extracted from the first-tier network. Double PSs and remaining single PSs with maximum NTM threshold of 0.60 and 0.72 respectively are derived especially on the two buildings. Thanks to the two-tier network applied, we don't need to use the spatiotemporal filtering to remove the APS. Then, we utilize a temporal highpass filtering, e.g. a Gaussian filter with a temporal threshold of 0.1 year in this study, to separating atmospheric artifacts from non-linear motions before their integrations. Finally, we derive the accumulated deformation time series with reference to the first SAR acquisition, allowing the monitoring and analysis of potential structural risks. 


\section{RESULTS AND DISCUSSIONS}

In order to understand the dynamic behaviour and the thermodynamics of building materials on the two skyscrapers in Shanghai Lujiazui Zone, we apply the Tomo-PSInSAR approach to quantitatively assess motion trends caused by thermal expansion, creep and shrinkage on the two buildings.

\subsection{Validation of Height Estimates}

Figure 3 shows the height map of the two skyscrapers estimated by the Tomo-PSInSAR approach. The two high-rise buildings are clearly visible in the height map. We observe that the estimated height of SWFC is approximately $490 \mathrm{~m}$, which indicates that the estimated height is in good agreement with the true height (see Table 1). However, the estimated height of Jin Mao Tower is approximately $383 \mathrm{~m}$ and there is a bias with the true height. The bias is most likely caused by that mistakenly introducing PSs comes from the spire of the tower into the surrounding structures. The validity of height estimates can validate the estimated thermal amplitude and linearly deformation velocity to some extent (Ferretti et al., 2000).

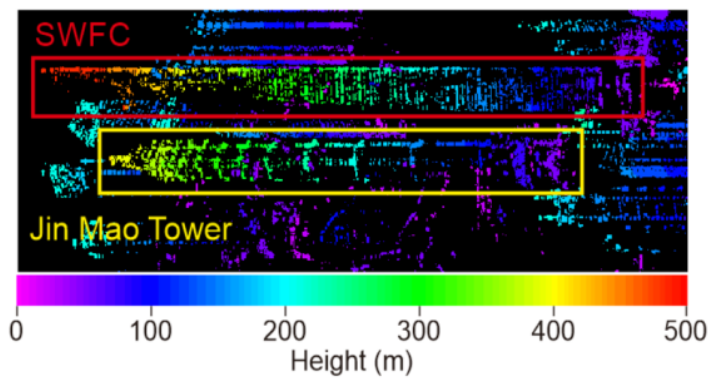

Figure 3. Height map of the two skyscrapers in Shanghai Luijiazui Zone estimated by the Tomo-PSInSAR. The red rectangle in the top figure denotes the SWFC, and the yellow rectangle denotes the Jin Mao Tower.

\subsection{The Effect of Thermal Expansion}

The extended 4-D SAR phase model is exploited to separate thermal expansion from total deformations. Figure 4 shows the thermal amplitude map of the two skyscrapers estimated by the Tomo-PSInSAR approach. We can see that the derived thermal amplitude is highly related to the building height. This relationship is most likely due to the accumulative effect of thermal expansion along the vertical direction (Crosetto et al., 2015). The deformation amplitude associated with changes in temperature is therefore largest at the top of buildings and is reduced to zero at the bottom. In our study, the height of SWFC ranges from 5 to $490 \mathrm{~m}$, and the thermal amplitude increases from $0.3 \mathrm{~mm} /{ }^{\circ} \mathrm{C}$ at $h=5 \mathrm{~m}$ to $4.6 \mathrm{~mm} /{ }^{\circ} \mathrm{C}$ at $h=490 \mathrm{~m}$, whereas the derived height of Jin Mao Tower ranges from 3.9 to $383 \mathrm{~m}$, and the thermal amplitude increases from $0.26 \mathrm{~mm} /{ }^{\circ} \mathrm{C}$ at $h=$ $3.9 \mathrm{~m}$ to $2.36 \mathrm{~mm} /{ }^{\circ} \mathrm{C}$ at $h=383 \mathrm{~m}$. Then, we calculate the gradient of thermal amplitude to be $0.0055 \mathrm{~mm} /{ }^{\circ} \mathrm{C}$ per meter for SWFC, and $0.0088 \mathrm{~mm} /{ }^{\circ} \mathrm{C}$ per meter for Jin Mao Tower, which implies that Jin Mao Tower has higher thermal expansion lever than SWFC. The reason is likely attributed to the different thermal expansion coefficients of the building materials. Finally, we derive the deformation time series of points P1 and P2 on SWFC and Jin Mao Tower due to thermal expansion as shown in Figure 5, which indicates a distinct seasonal trend associated with changes in temperature in time series.

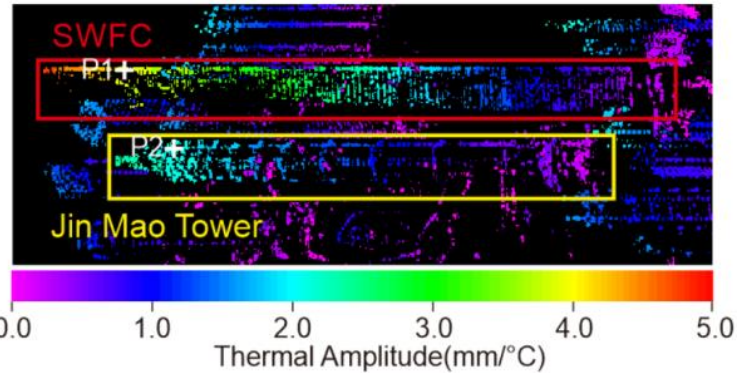

Figure 4. Thermal amplitude map of the two skyscrapers in Shanghai Lujiazui Zone estimated by theTomo-PSInSAR. The red rectangle in the top figure denotes the SWFC, and the yellow rectangle denotes the Jin Mao Tower.

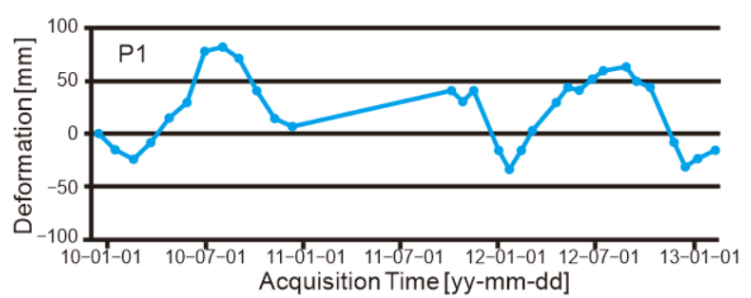

(a)

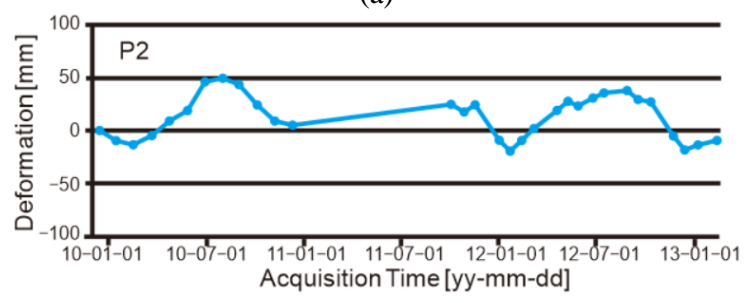

(b)

Figure 5. Deformation time series of PS points P1 and P2 due to thermal expansion, are indicated by white crosses located in two skyscrapers (a) SWFC and (b) Jin Mao Tower in Shanghai Lujiazui Zone.

\subsection{Deformation due to Creep and Shrinkage}

Figure 6 shows the deformation velocity map of the two skyscrapers estimated by the Tomo-PSInSAR approach. We can observe visually that the velocity of PSs in SWFC is much higher than the velocity of PSs in the surrounding ground surface, meaning that SWFC is subject to deformation during the new built period, causing the building to move away from the sensor. In addition, we also notice the deformation is a height-independent movement, i.e., at higher positions of the building move away from the sensor more quickly than those at lower positions, and the possible reason may be wall shortening under the combined effects of concrete creep and shrinkage. To study the evolution of creep and shrinkage, we retrieve the deformation time series of point P1 on the SWFC after eliminating the influence of thermal expansion as shown in Figure 7(a), which indicates that the deformation rate caused by creep and shrinkage are largest at the beginning and gradually decrease, and finally attain a steady state as time approaches infinity. On the contrary, the linear deformation velocity map and the deformation time serious of point P2 in Jin Mao Tower shown in Figure 7(b) implies that Jin Mao Tower is almost steady and does not decline during this period. This is because Jin Mao Tower is an old built building, which is not influenced by creep and shrinkage as the load is relaxed and dehydration proceeds. 


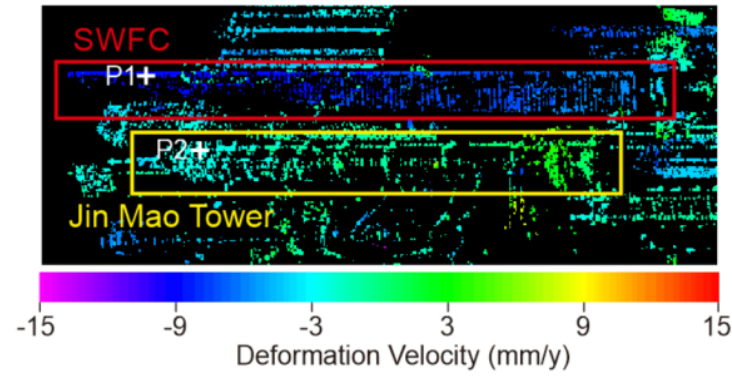

Figure 6. Deformation velocity map of the two skyscrapers in Shanghai Lujiazui Zone estimated by the Tomo-PSInSAR. The red rectangle in the top figure denotes SWFC, and the yellow rectangle denotes Jin Mao Tower.

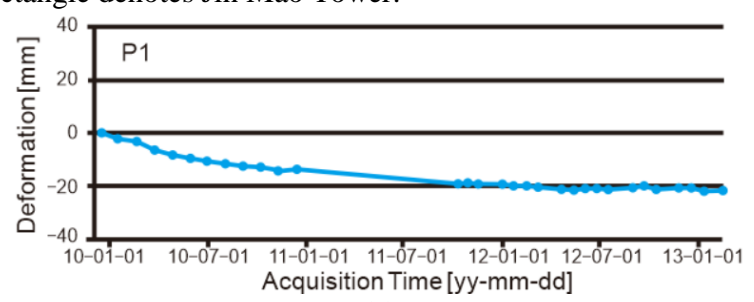

(a)

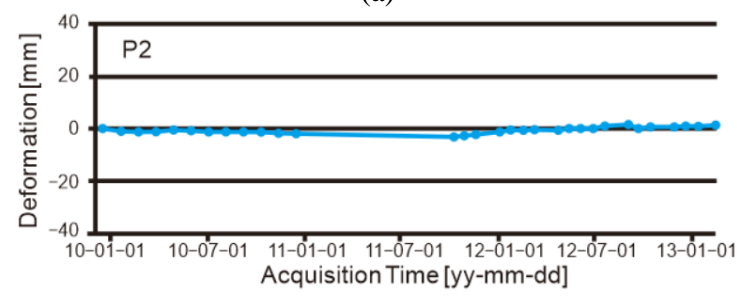

(b)

Figure 7. Deformation time series of PS points P1 and P2 due to creep and shrinkage are indicated by white crosses located in two skyscrapers (a) SWFC and (b) Jin Mao Tower in Shanghai Lujiazui Zone.

\section{CONCLUSIONS}

In this study, we utilize a Tomo-PSInSAR approach for monitoring the deformation performances of high-rise buildings, i.e. SWFC and Jin Mao Tower, in Shanghai Lujiazui Zone. For the purpose of this study, we use 31 Stripmap acquisitions from TerraSAR-X missions, spanning from December 2009 to February 2013. We use an extended 4-D SAR phase model to estimate three parameters (height, deformation velocity, and thermal amplitude) simultaneously and apply a two-tier network strategy to detect single and double PSs with no need for preliminary removal of the APS in the study area. The thermal amplitude map demonstrates that the derived thermal amplitude of two high-rise buildings both dilate and contract periodically, which is highly related to the building height due to the upward accumulative effect of thermal expansion. The linear deformation velocity map reveals that SWFC is subject to deformation during the new built period due to creep and shrinkage, which is height-dependent movements in the linear velocity map. On the contrary, the linear deformation velocity map shows that Jin Mao Tower is almost steady and does not decline during this period. This is because it is an old built building, which is not influenced by creep and shrinkage as the load is relaxed and dehydration proceeds. This study underlines the potential of the Tomo-PSInSAR solution for the monitoring deformation performance of high-rise buildings, which offers a quantitative indicator to local authorities and planners for assessing potential damages.

\section{ACKNOWLEDGEMENTS (OPTIONAL)}

This research was jointly supported by the National Natural Science Foundation of China (Grant No. 41501461), the sponsorship of Jiangsu Overseas Research and Training Program for University Prominent Young and Middle-aged Teachers and Presidents.

\section{REFERENCES}

Ferretti, A., Prati, C., Rocca, F., 2000. Nonlinear subsidence rate estimation using permanent scatterers in differential SAR interferometry. IEEE Transactions on Geoscience and Remote Sensing, 38 (5), pp. 2202-2212.

Huber, P.J., 1964. Robust estimation of a location parameter. Annals of Mathematical Statistics, 35 (1), 73-101.

Jiang, Y., Liao, M., Wang, H., Zhang, L., Balz, T., 2016. Deformation Monitoring and Analysis of the Geological Environment of Pudong International Airport with Persistent Scatterer SAR Interferometry. Remote Sensing, 8(12), pp. 1021.

Ma, P., Lin, H., Lan, H., Chen, F., 2015. Multi-dimensional SAR tomography for monitoring the deformation of newly built concrete buildings. ISPRS Journal of Photogrammetry and Remote Sensing, 106 (2015), pp. 118-128.

Ma, P., Lin, H., 2016. Robust detection of single and double persistent scatterers in urban built environments. IEEE Transactions on Geoscience and Remote Sensing, 54 (4), pp. 2124-2139.

Monserrat, O., Crosetto, M., Cuevas, M., Crippa, B., 2011. The thermal expansion component of persistent scatterer interferometry observations. IEEE Geoscience and Remote Sensing Letters, 8 (5), pp. 864-868.

Perissin, D., Wang, Z., Lin, H., 2012. Shanghai subway tunnels and highways monitoring through cosmo-skymed persistent scatterers. ISPRS Journal of Photogrammetry and Remote Sensing, 73 (2012), pp. 58-67.

Wu, J., Hu, F., 2017. Monitoring Ground Subsidence Along the Shanghai Maglev Zone Using TerraSAR-X Images. IEEE Geoscience and Remote Sensing Letters, 14 (1), pp. 117-121. 